\title{
Shadowing Corrections and the Precise Determination of Electroweak Parameters in Neutrino-Nucleon Scattering
}

\author{
Gerald A. Miller \\ University of Washington Seattle, WA 98195-1560 \\ A. W. Thomas \\ Department of Physics and Mathematical Physics, and Special Research Centre for the \\ Subatomic Structure of Matter \\ Univ. of Adelaide, Adelaide 5005, Australia
}

\begin{abstract}
A systematic error in the extraction of $\sin ^{2} \theta_{W}$ from nuclear deep inelastic scattering of neutrinos and antineutrinos arises from higher-twist effects arising from nuclear shadowing. We explain that these effects cause a correction to the results of the recently reported significant deviation from the Standard Model that is potentially as large as the deviation claimed, and of a sign that cannot be determined without an extremely careful study of the data set used to model the input parton distribution functions.
\end{abstract}

In a recent and stimulating paper [1], the $\mathrm{NuTeV}$ collaboration reported a determination of $\sin ^{2} \theta_{W}$, based on a comparison of charged and neutral current neutrino interactions with a nuclear target $(\mathrm{Fe})$, which differs from the Standard Model prediction by three standard deviations. In view of the importance of such a result it is vital that the sources of systematic error be clearly identified and examined. Here we explain that there is a nuclear correction, arising from the higher-twist effects of nuclear shadowing [2,3], for which no allowance has been made in the NuTeV analysis. This correction may well be of the same size as the reported deviation.

The measurement under consideration involves the separate measurements of the ratios of neutral current (NC) to charged current (CC) cross sections on Fe for $\nu$ and $\bar{\nu}$. The best values of $\sin ^{2} \theta_{W}$ and $\rho_{0}$ are extracted from the precisely determined ratios. But the nuclear effects must be removed. Because a substantial fraction of the $\mathrm{NuTeV}$ data in the region $x$ below 0.1 is at relatively low $Q^{2}$ (even though the average $Q^{2}$ is $16 \mathrm{GeV}^{2}$ ), one expects a significant shadowing contribution from vector meson dominance (VMD) [4,5], which is of higher twist. As explained by Boros et al. [2,3], the effect of the VMD contribution to nuclear shadowing in neutrino interactions is substantial, and leads to a reduction of the $\mathrm{CC} \nu$ cross section by about $50 \%$ compared with the reduction found for photons. (Briefly, the VMD contribution to shadowing is dominated by the $\rho$ meson and $f_{\rho^{+}}^{2}=2 f_{\rho^{0}}^{2}$, whereas the $\mathrm{CC}$ to photon cross sections are in the ratio 18/5.) Together with a full NLO analysis of the data, this was important in reconciling the NuTeV and NMC data without any need for substantial charge symmetry violation of the parton distributions [6]. A recent re-examination of the role of vector meson dominance in nuclear shadowing at low $Q^{2}$ finds that models (such as that used here) which incorporate both vector meson and partonic mechanisms are consistent with both the magnitude and the $Q^{2}$ slope of the shadowing data [7].

For present purposes we need also to consider this higher-twist effect of shadowing of vector mesons for $\bar{\nu}$ interactions. These involve predominantly anti-quarks and the shadowing effect is relatively larger by a factor of three or so. However, the VMD contribution to shadowing for neutral current interactions is $1 / 2$ of that for charged current interactions because $Z$ conversion to 
a $\rho^{0}$ occurs with a factor of $(1 / 2)$ of that for $W^{+} \rightarrow \rho^{+}$.

Let us examine how these differences in shadowing effects influence the extraction of $\sin ^{2} \theta_{W}$. Suppose the nuclear cross section for NC interactions of neutrinos is larger than that for CC interactions by a factor of $1+\frac{1}{2} \epsilon$ and that the one for anti-neutrinos is larger by a factor of $1+\frac{1}{2} \bar{\epsilon}$, with $\bar{\epsilon}$ expected to be substantially larger than $\epsilon$. Then the nuclear ratios of neutral current (NC) to charged current $(\mathrm{CC})$ cross sections are

$$
\begin{aligned}
& R_{A}^{\nu} \equiv \frac{\sigma_{A}(\nu A \rightarrow \nu X)}{\sigma_{A}\left(\nu A \rightarrow \ell^{-} X\right)}=\frac{\sigma(\nu N \rightarrow \nu X)}{\sigma\left(\nu N \rightarrow \ell^{-} X\right)}\left(1+\frac{1}{2} \epsilon\right)=\left(1+\frac{1}{2} \epsilon\right)\left(g_{L}^{2}+r g_{R}^{2}\right) \\
& R_{A}^{(\bar{\nu})} \equiv \frac{\sigma_{A}(\bar{\nu} A \rightarrow \bar{\nu} X)}{\sigma_{A}\left(\bar{\nu} A \rightarrow \ell^{(+)} X\right)}=\left(1+\frac{1}{2} \bar{\epsilon}\right)\left(g_{L}^{2}+r^{(-1)} g_{R}^{2}\right)
\end{aligned}
$$

where $r=\sigma\left(\bar{\nu} N \rightarrow \ell^{+} X\right) / \sigma\left(\nu N \rightarrow \ell^{-} X\right), r_{A}=r\left(1+\frac{1}{2} \epsilon\right) /\left(1+\frac{1}{2} \bar{\epsilon}\right), g_{L}^{2}=1 / 2-\sin ^{2} \theta_{W}+$ $5 / 9 \sin ^{4} \theta_{W}$ and $g_{R}^{2}=5 / 9 \sin ^{4} \theta_{W}$. Equations (1) and (2) tell us that the nuclear-shadowing corrections for $R_{A}^{\nu}$ and $R_{A}^{(\bar{\nu})}$ are not the same, and that the extraction of $\sin ^{2} \theta_{W}$ requires the separate knowledge of $\epsilon$ and $\bar{\epsilon}$.

A detailed analysis of the NuTeV data requires that one model the ratios $R_{A}^{\nu}$ and $R_{A}^{(\bar{\nu})}$ at a required accuracy of a fraction of a percent. This, in turn, requires an even more accurate knowledge of both the quark and antiquark parton distribution functions (pdfs). In general, the pdfs are derived from a global analysis data from electron/muon, $\mathrm{CC}$ neutrino and NC neutrino deep inelastic scattering on protons, deuterons and nuclei. The range of $Q^{2}$, particularly at low $x(x \leq 0.1)$ can be quite low. Higher twist shadowing corrections are almost universally ignored in global determinations of the pdfs. This is certainly the case for the pdfs used by NuTeV. Since the VMD shadowing corrections are different for electrons, CC neutrino scattering and $\mathrm{NC}$ neutrino scattering, the pdfs resulting from such a global analysis are at best an approximation to the true ones, with unknown systematic errors. Worse, one cannot simply add a shadowing correction to a simulation based on such global pdfs as even the sign of the correction will depend on the particular data sets included in the analysis. Of course, the systematic errors encountered will not be serious for most purposes. However, in this case, where the signal of a deviation from the Standard Model is at the percent level, one must control this potential source of error extremely carefully.

We cannot undo the global analysis of pdfs by the NuTeV collaboration. However, we can make an estimate of the order of magnitude of the shadowing corrections using Eqs. (1) and (2). The quantity $\epsilon$ is given as a function of $x$ for $Q^{2}=5 \mathrm{GeV}^{2}$ as the dashed curve of Fig $3 \mathrm{~b}$. in Ref. [2]. One needs to take the deviation between dashed curve and unity. Thus, e.g. $\epsilon\left(x, Q^{2}=\right.$ $\left.5 \mathrm{GeV}^{2}\right) \approx 0.041$ at $x=10^{-2}$. For other values of $Q^{2}$, one may use: $\epsilon\left(x, Q^{2}\right) \approx \epsilon\left(x, Q^{2}=\right.$ $\left.5 \mathrm{GeV}^{2}\right)\left(\frac{m_{\rho}^{2}+5 \mathrm{GeV}^{2}}{m_{\rho}^{2}+Q^{2}}\right)^{2}$. Furthermore $\bar{\epsilon} / \epsilon \approx F_{2}^{D}(\nu) / F_{2}^{D}(\bar{\nu}) \approx 2$. Using a typical value of $Q^{2} \approx$ $10 \mathrm{GeV}^{2}$ and range of $x \approx 0.05$ of the NuTeV experiment leads to the values $\epsilon=0.006$ and $\bar{\epsilon}=0.012$ [8]. Thus the nuclear corrected ratios $R_{A}^{\nu}$ and $R_{A}^{\bar{\nu}}$ would be smaller than those reported in Ref. [1], by 0.003 and 0.006 respectively. That these numbers represent important corrections can be seen immediately by examining the sources of errors reported in Ref. [1]. The total error for $R_{A}^{\nu}$ is reported as 0.0013 and that for $R_{A}^{\bar{\nu}}$ as 0.00272 . The effects of shadowing are larger than these quoted errors by a factor of two or three! It is clear that any analysis of nuclear data aimed at determining $\sin ^{2} \theta_{W}$ must account for nuclear shadowing. But this has not been done in Ref. [1].

It is necessary to carry out a more refined analysis of the data which properly incorporates the high twist components of nuclear shadowing, starting with the pdfs themselves. Such an 
analysis should also take into account the experimental acceptances as a function of $x, y$ and $Q^{2}$. Alternatively, one could drastically reduce the VMD contribution by restricting the data set to events with $Q^{2}>5 \mathrm{GeV}^{2}$ - although we understand that this may present difficulties for NC events.

We find that the size of the shadowing effects is substantial and should be incorporated in the experimental analysis. It is true that the simplest estimate of this effect (ignoring the effects on the pdfs themselves, which was discussed above) is opposite to that required to explain the deviation from the Standard Model. Thus it may be that the deviation from the Standard Model could be even larger than reported in Ref. [1].

Finally, we note that several other effects that tend to reduce the discrepancy have been reported. The influence of charge symmetry breaking, arising from the mass difference between up and down quarks [9], accounts for about a third to a half of the deviation between NuTeV's value of $\sin ^{2} \theta_{W}$ and that of the Standard Model in a model-independent manner [10] . Furthermore, it has been known for more than 20 years that parton distributions of nucleons bound in nuclear matter differ from those of free nucleons. Such effects [11] still present a considerable challenge to our understanding of nonperturbative QCD and it is not inconceivable that they could eventually account for the entire deviation of $\sin ^{2} \theta_{W}$.

It seems clear that the extraction of the the value of $\sin ^{2} \theta_{W}$ from neutrino-nuclear interactions involves handling several different types of corrections of different signs, including some that are difficult evaluate with precision. The situation here may well be similar to many in strong interaction physics, in which a "cocktail" of effects is required [12]. Considering that possible explanations in terms of new physics are not compelling [13], considerable efforts must be applied before concluding that the NuTeV result really demonstrates a deficiency of the Standard Model.

We thank the USDOE for partial support and M. Ramsey-Musolf for a useful remark. This work was also supported by the Australian Research Council.

[1] G. P. Zeller et al., Phys. Rev. Lett. 88, 091802 (2002).

[2] C. Boros, J. T. Londergan and A. W. Thomas, Phys. Rev. D 59, 074021 (1999);

[3] Phys. Rev. D 58, 114030 (1998).

[4] J. Kwiecinski and B. Badelek, Phys. Lett. B 208, 508 (1988).

[5] W. Melnitchouk and A. W. Thomas, Phys. Rev. C 52, 3373 (1995).

[6] C. Boros, F. M. Steffens, J. T. Londergan and A. W. Thomas, Phys. Lett. B 468, 161 (1999).

[7] W. Melnitchouk and A. W. Thomas, Phys. Rev. C 67, 038201 (2003) [arXiv:hep-ex/0208016].

[8] For accuracy, one needs to know the fraction of the NuTeV events obtained at low $x$ and $Q^{2}$. Using $Q^{2}=10 \mathrm{GeV}^{2}$ reflects a compromise between using using the average value $\sim 20 \mathrm{GeV}^{2}$ where the shadowing is completely absent, and the lower values characteristic of the low $x$ region where shadowing is important.

[9] G. A. Miller, B. M. Nefkens and I. Slaus, Phys. Rept. 194, 1 (1990).

[10] E. Sather, Phys. Lett. B 274, 433 (1992); E. N. Rodionov, A. W. Thomas and J. T. Londergan, Mod. Phys. Lett. A 9, 1799 (1994); J. T. Londergan and A. W. Thomas, Phys. Rev. D 67, 111901 (2003) [arXiv:hep-ph/0303155]; J. T. Londergan and A. W. Thomas, Phys. Lett. B 558, 132 (2003) [arXiv:hep-ph/0301147]. 
[11] S. Kovalenko, I. Schmidt and J. J. Yang, Phys. Lett. B 546, 68 (2002) [arXiv:hep-ph/0207158]; S. Kumano, Phys. Rev. D 66, 111301 (2002) [arXiv:hep-ph/0209200]. S. A. Kulagin, Phys. Rev. D 67, 091301 (2003) [arXiv:hep-ph/0301045].

[12] G. Taubes, "Nobel Dreams:Power Deceit and the Ultimate Experiment", Random House NY, NY (1993)

[13] S. Davidson, J. Phys. G 29, 2001 (2003) [arXiv:hep-ph/0209316]. 